\title{
Ein faszinierender Blick über den großen Teich
}

\author{
Bericht über den 4. internationalen Fascia Research Congress (FRC) in Washington
}

Doris Börner

Nach den drei sehr erfolgreichen Kongressen von 2007 in Boston, 2009 in Amsterdam und 2012 in Vancouver fand im September 2015 in Washington der 4. internationale Fascia Research Congress (FRC) unter der Schirmherrschaft der Ida Rolf Foundation statt. Mehr als 800 Teilnehmer aus 35 Ländern fanden sich zu diesem Anlass auf diesem Kongress ein. Ziel des Kongresses war es auch in diesem Jahr, den Vertretern der Grundlagenforschung ebenso wie den im Gesundheitsbereich arbeitenden Praktikern eine Plattform zum Erfahrungsaustausch Rund um das Thema Faszien zu bieten.

\section{Eine interdisziplinäre Zusammenarbeit}

Zum ersten Mal wurden - neben Tiermodell-Erfahrungen aus der Grundlagenforschung - auch veterinärmedizinische Aspekte der Faszienforschung auf dem Kongress diskutiert. Interdisziplinäre Zusammenarbeit ergibt Sinn: So werden neue Einsichten ausgetauscht, um bisherige Vorstellungen und Therapiemethoden praxisnah zu evaluieren, Ressourcen zu sparen, wissenschaftliche Arbeit besser zu fokussieren und ihre Entwicklung erfolgreicher zu machen. Mehr

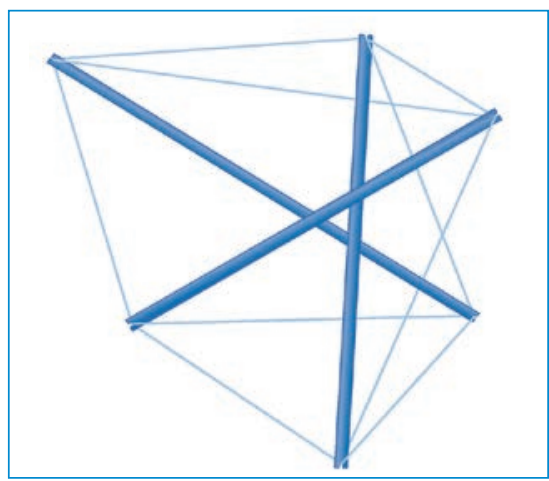

Abb. 1 Schematische Darstellung des Tensegrity-Modells. (Quelle: Könneker H, Reiter U. Osteopathie in der Kleintierpraxis. 1. Aufl. Stuttgart: Sonntag; 2010) als 180 peer-reviewed Abstracts wurden dem Kongresskomitee eingereicht und bildeten eine herausragende Basis zur Gestaltung des Fachprogramms.

\section{Entwicklung der Faszienforschung}

Seit der Publikation des Kongressberichts von 2007 wurden mehr als 10000 Artikel in 19 Sprachen in peer-reviewed Journals veröffentlicht, die sich mit dem Thema Faszienforschung beschäftigen. Diese Entwicklung ist nicht zuletzt der Tatsache zu verdanken, dass in letzter Zeit viele anatomische und histologische Strukturen der Faszie re-evaluiert wurden. Gleichzeitig ermöglichen neu entwickelte Untersuchungsmethoden wie hochauflösender Ultraschall und bioelektrische Impedanz die Untersuchung des Fasziengewebes in vivo. Auf dieser Basis ist nun mehr die Darstellung und Beschreibung der Effekte manueller Methoden möglich geworden, was ein neues Kapitel in der Faszienforschung bei Mensch und Tier eröffnet.

\section{Faszien: Was sind sie genau?}

Was genau ist denn nun eigentlich diese Bindegewebsmatrix, mit der sich zurzeit so viele Wissenschaftler und Forscher beschäftigen? Im Jahr 2007 wurde von Finley und Schleip die erste Definition anlässlich des 1. Faszien-Kongresses vorgeschlagen, die 2009 von Huijing und Langevin weiterentwickelt wurde. Die Faszie beschreibt demnach kurz gesagt „die Weichteilgewebeanteile des den (menschlichen) Körper durchziehenden Binde- und Stützgewebeapparats“. Anders - eher funktionell ausgedrückt sind es die kollagenhaltigen Fasern, die als Grundlage für das Kraft-Übertragungssystem des Körpers via Zugspannung dienen. Die Vorlage dieser Vorstel- lung der dynamischen Verbindung über Zugspannung entstammt dem „Tensegrity"-Konzept - der Kombination aus Spannung (Tension) und Zusammenhalt (Integrity) - dessen Ursprung in der Architektur erarbeitet wurde ( $\triangleright$ Abb. 1).

Faszien beschreiben somit nicht nur die Gewebe, die wir auf den ersten Blick als solche einordnen würden (wie z.B. Septen, Gelenkkapseln und Aponeurosen), sondern ebenso andere verdichtete Gewebe im Spannungsnetzwerk wie z.B. das bronchiale Bindegewebe oder die fibrinöse äußere Schicht der Bandscheiben. Um den neuen Erkenntnissen der funktionellen resp. anatomischen Ambivalenz der Faszien Rechnung zu tragen, wurde von dem Federal Committee of Anatomical Terminology (FCAT) nun in diesem Jahr im Rahmen des Kongresses die Anfrage geäußert, für die neue Ausgabe der Terminologia Anatomica 2016 einen neuen Begriff der Faszie festzulegen.

\section{Funktion und Bedeutung der Faszien}

Angesichts der Tragweite der Erkenntnis über die Funktion der Faszien im Organismus wird man sicher auch nicht darum herumkommen, die Funktion und Bedeutung der Faszien auch in der veterinärmedizinischen Anatomie und Praxis neu einzuordnen. Die westliche Anatomie ähnelte bisher im Wesentlichen eher einer „Skalpell Anatomie“, als dass sie funktionelle Komponenten berücksichtigt. Ein Muskel oder Organ stellt sich eben auch viel eindeutiger dar, wenn man ihn von jeglichen Verbindungen freipräpariert. Dass dabei auch ein sehr großer Anteil seiner Funktion verloren geht, wird durch die neuen Darstellungsmethoden aus der Anatomie sehr klar. Ein beeindruckendes Beispiel ist der 2015 erschienene Atlas über das Fasziennetz des Menschen von Frau Dr. Carla 
Stecco von der Universität in Padua, in dem der Mensch wie in einem Taucheranzug aus Bindegewebe dargestellt wird [8]. Bemerkenswerterweise handelt es sich auch in der Humanmedizin um den ersten Atlas dieser Art.

Funktionelle Untersuchungen am Tier, wie sie beispielsweise von Prof. Dr. Martin S. Fischer in Jena durchgeführt wurden, sind ein eindeutiger Hinweis darauf, dass wir unser bisheriges Verständnis der funktionellen Anatomie bzw. der funktionellen Neurologie auch beim Tier überdenken müssen. In dem neu erschienenen Lehrbuch zur Lahmheitsdiagnostik von Daniel Koch und Martin S. Fischer haben diese neuen Überlegungen bereits Berücksichtigung gefunden. Ebenso gilt es auch, Therapiemethoden entsprechend den sich immer wieder neu ergebenden Erkenntnissen anzupassen, denn um mit den Worten von Tom Myers zu sprechen: „Fakten haben doch oft eine sehr kurze Halbwertszeit“.

Entsprechend einer vorgestellten Studie beispielsweise, bei der der Einfluss von mechanischen Therapien auf die Heilung chronischer Tendinopathien untersucht wurde, konnte dargestellt werden, dass ein kontrolliertes Bewegungsregime auf dem Laufband eine direkte Effektivität von mechanischen Stimuli auf den Heilungsverlauf einer Tendinopathie hat. Diese Untersuchung unterstützt die Arbeitshypothese, dass Fibroblasten in der Matrix eine zentrale Rolle spielen. Abhängig von der Bewegung entstehen offenbar unterschiedliche Strukturen. Für die Klinik bedeutet dies, dass der Heilungsverlauf zu einer belastbaren Architektur direkt abhängig von physikalischen Einflüssen zu sein scheint, die auf das Gewebe einwirken. Zellen in der extrazellulären Matrix werden durch mechanische Faktoren beeinflusst, die das Proteinmilieu verändern. Dieses wird von Entzündungsmediatoren und dem Krankheitsprozess selbst moduliert, aber auch von den Übungen im Rahmen der Rehabilitation. Ein Übungsplan kann demnach degenerativen Prozessen vorbeugen, wobei insbesondere die zentrische versus exzentrische Belastung der Muskulatur eine zentrale Rolle zu spielen scheint. Dies ist für die Erstellung eines
Rehabilitationsplans bei Sehnenverletzungen nicht gerade unwesentlich: Vielleicht sind Boxenruhe und Leinenzwang doch nicht immer die beste Antwort auf alle Probleme des Bewegungsapparats.

\section{Faszien und Schmerzen}

Faszien sind offenbar ein sehr wichtiges „Organ“ mit sensiblen Eigenschaften, die darum auch in der Propriozeption eine große Rolle zu spielen scheinen. Eine Stimulation der Faszie, einschließlich der thorakolumbalen Faszie (TLF), verursacht Schmerz. In einer Untersuchung der TLF bei Ratten wurden die Existenz nozizeptiver Fasern in Faszien sowie der Einfluss der Entzündung auf diese Fasern erforscht. Zu diesem Zweck wurden die Länge und die Anzahl der freien Nervendigungen anhand von Antikörberverbindungen an die charakteristischen Axone oder Membranen dieser Fasern untersucht. SP, CGRP-positive und TRPV1Moleküle (ein charakteristisches nozizeptives Rezeptormolekül) wurden in dieser Studie nachgewiesen.

Faszien enthalten demnach Nozizeptoren, wobei sich diese nach der induzierten Entzündung nicht nur in der oberen, sondern auch in der tiefen Schicht der Faszie befinden. Vermutlich ist es also nicht nur der Muskel, der schmerzt, sondern auch - vielleicht sogar hauptsächlich - die Faszie. Aus einer Studie an gesunden Patienten hat sich ergeben, dass die Injektion eines Analgetikums in die Faszie schmerzhafter als die Injektion in den Muskelbauch ist. Der Input, der von der Faszie an das Rückenmark gegeben wird, kann dazu zu segmentaler, lang anhaltender Sensibilisierung der Schmerzempfindlichkeit führen.

\section{Ein Spaziergang unter der Haut}

Um einen weiteren staunenden Einblick in die filigrane Architektur der Natur in vivo zu bekommen, hat Jean Claude Guimberteau einzigartige endoskopische Aufnahmen von mikroanatomischen Strukturen in lebendem Gewebe angefertigt und gezeigt, wie diese das feinmaschige Netzwerk den gesamten Organismus durchziehen (Strolling under the skin, [4]). Plastische und elastische Strukturen in dem Gewebe bleiben von außen unbemerkt, denn eben das pas- siert, wenn eine Hautfalte aufgezogen wird, um dann wieder in ihre Ausgangsposition zurückzuschnellen. Das Gewebe verhält sich wie ein Gummiband, das in seine Ausgangsform zurückschnellt, im Gegensatz zu einer Delle, die in einer Plastiktüte verbleibt, wenn man diese mit dem Finger verformt.

Aber wie verändern wir durch Eingriffe diese Fähigkeit der Resilienz? Bei chirurgischen Eingriffen werden die Zugänge präpariert, aber diese Zugänge existieren nicht in lebendem Gewebe, denn Gewebekontinuität ist total, wie in dem Faszien-Atlas von Frau Carla Stecco eindrücklich dargestellt wird [8]. Der wissende, umsichtige Umgang bei der Planung und Durchführung von Operationen hat damit demnach einen großen Einfluss auf den postoperativen Heilungsprozess.

\section{Funktionelle Anatomie und Bewegung}

Über das Verständnis von funktioneller Anatomie und Bewegung bleibt noch viel zu lernen. Die ersten Früchte der interdisziplinären Arbeit in der Faszienforschung finden sich beispielsweise in einer Studie aus Dänemark, bei der die funktionell verbindenden Linien und Strukturen nach dem Vorbild von Tom Myers „Anatomy Trains“ bei 26 Pferden untersucht wurden [1]. Ein Unterschied zwischen Zwei- und Vierbeinern war zu 
erwarten, aber das Verständnis von Lokomotion muss nach diesen ersten Erkenntnissen wohl auch bei den Vierbeinern überdacht und eine eher holistische Annäherung an Erkrankungen in Betracht gezogen werden, die über das „Ansatz-Ursprung-Funktionsmodell“ eines Muskels hinausgeht.

Online zu finden unter

http://dx.doi.org/10.1055/s-0041-109708

\section{Literatur}

1 Elbrønd VS, Schultz RM. Myofascia - the unexplored tissue: Myofascial kinetic lines in horses, a model for describing locomotion using comparative dissection studies derived from human lines. Medical Reseach Archives 2015; 3

2 Fascia Research 2015 - State of the Art. Proceedings of the Fascia Research Congress 2015: Washington, DC, 19.-20. September

\section{Fazit}

Der Fascia Research Congress bedeutet interdisziplinäre Zusammenarbeit auf höchstem Niveau und ein Zugewinn an Erkenntnissen, die auch in tiermedizinischen Überlegungen miteinzubeziehen sind. Was alle Teilnehmer unabhängig vom Fachbereich verbindet - und sicher auch in Zukunft verbinden wird - findet sich in einer Bemerkung von Robert Schleip im Rahmen des Faszienforums in Hamburg: „Uns vereinigt nicht das Wissen, sondern das Wissen-Wollen“.
3 Fischer MS, Lilje KE. Hunde in Bewegung. Stuttgart: Kosmos; 2011

4 Guimberteau JC. Strolling under the skin (2014). Im Internet: https://www.youtube. com/watch?v=eWOlvOVKDxE; Stand: 18.11.2015

5 Koch D, Fischer MS. Lahmheitsuntersuchung beim Hund. Funktionelle Anatomie, Diagnostik und Therapie. Stuttgart: Enke; 2015

6 Myers TW. Anatomy Trains - Myofasziale Leitbahnen. 3. Aufl. München: Urban \& Fischer/Elsevier; 2014
7 Schleip R, Findley TW, Chaitow L, Hujiing PA. Lehrbuch Faszien. München: Urban \& Fischer/ Elsevier; 2014

8 Stecco C. Functional Atlas of the Human Fascial System. Churchill Livingston Elsevier; 2015

\section{Dr. med. vet. Doris Börner}

iuvet - Praxis für funktionelle Neurologie, Chiropraxis, Akupunktur und TCVM

Danziger Str. 9d

22869 Schenefeld

info@iuvet.de

www.iuvet.de 